\title{
Velocidade da água e a distribuição de larvas e pupas de Chirostilbia pertinax (Kollar) (Diptera, Simuliidae) e macroinvertebrados associados
}

\author{
José Eloy dos Santos Jr. ${ }^{1}$, Milton Norberto Strieder ${ }^{1}$, Gelson Luiz Fiorentin ${ }^{1} \&$ Ulisses Gaspar Neiss ${ }^{1}$
}

${ }^{1}$ Laboratório de Entomologia, Ciências da Saúde, Universidade do Vale do Rio dos Sinos. Av. Unisinos 950, 93022-000 São Leopoldo-RS, Brasil. strieder@unisinos.br

\begin{abstract}
Water velocity and the distribution of larvae and pupae of Chirostilbia pertinax (Kollar) (Diptera, Simuliidae) and associated macroinvertebrates. The objective of the current study was to evaluate the distribution of the immature stages of Chirostilbia pertinax (Kollar, 1832) and associated bentic macroinvertebrates in relation to water velocity. This study was carried out on the upper course of Sinos river, in Rio Grande do Sul. Seasonal collections of larvae and pupae of black flies and associated benthic fauna were carried out at five places. In all sampling sites artificial substrates were installed, remaining submersed for 14 days in places of great water flow. Before removing the substrates the flow speeds on the collection sites were measured. To assess the distinct distribution patterns of the benthic fauna, the flow speed was divided in three classes: slow $(0.20-0.63 \mathrm{~m} / \mathrm{s})$, medium $(0.64-1.07 \mathrm{~m} / \mathrm{s})$ and fast $(1.08-1.50 \mathrm{~m} / \mathrm{s})$. A total of 39,598 specimens were collected, from which 33,418 were black flies and 6,180 associated macroinvertebrates. From the total black flies, 5,704 were in the last larval instar and pupae from which 828 were $C$. pertinax. In the distribution of this species $30.8 \%$ occurred in slow flow, $24.9 \%$ in medium flow and $44.3 \%$ in fast flow. The abundance of C. pertinax is inversely related to the predatory macroinvertebrates $\left(\mathrm{GLM}, \mathrm{R}^{2}=0.207 ; \mathrm{F}{ }_{2.50}=6.536 ; \mathrm{P}=0.003\right)$. These results demonstrate that the immature stages of $C$. pertinax have a tendency to occupy microhabitats with less predator occurrence.
\end{abstract}

KEYWORDS. Benthic macroinvertebrates; black flies; C. pertinax; water flow speed.

RESUMO. Velocidade da água e a distribuição de larvas e pupas de Chirostilbia pertinax (Kollar) (Diptera, Simuliidae) e macroinvertebrados associados. O presente trabalho objetivou investigar a relação entre a velocidade da água e a distribuição dos estágios imaturos de Chirostilbia pertinax (Kollar, 1832) e os macroinvertebrados bentônicos associados. As coletas foram realizadas em cinco pontos, no trecho superior do Rio dos Sinos, no Estado do Rio Grande do Sul. A fauna bentônica foi amostrada com substratos artificiais, que permaneceram instalados nos locais de maior corenteza d'água por períodos de 14 dias. Antes do recolhimento dos substratos foram realizadas medidas de velocidade d'água no local da sua instalação. Essas medidas foram agrupadas em três classes: baixa $(0,20$ a $0,63 \mathrm{~m} / \mathrm{s})$, média $(0,64$ a $1,07 \mathrm{~m} / \mathrm{s})$ e alta $(1,08$ a $1,50 \mathrm{~m} / \mathrm{s})$ para posterior relação com a distribuição da fauna coletada. Para avaliar a influência da velocidade de água sobre a distribuição dos macroinvertebrados e a abundância de $C$. pertinax foi realizada uma regressão múltipla. As coletas resultaram em 39.598 indivíduos, sendo 33.418 simulídeos e 6.180 macroinvertebrados associados. Entre os simulídeos 5.704 espécimes correspondem a larvas do último ínstar e pupas, dos quais 828 são de $C$. pertinax. Quanto à distribuição dessa espécie, 30,8\% ocorreram na classe de baixa velocidade d'água, 24,9\% na velocidade média e 44,3\% na alta velocidade. Foi observado que a abundância de $C$. petinax aumenta à medida que os macroinvertebrados predadores diminuem e os organismos não predadores aumentam $\left(\mathrm{GLM}, \mathrm{R}^{2}=0,207 ; \mathrm{F}_{2.50}=6,536 ; \mathrm{P}=0,003\right)$. Esses resultados revelam que os imaturos de $C$. pertinax têm uma tendência em ocupar um microhábitat com menor ocorrência de predadores.

PALAVRAS-CHAVES. Borrachudos; C. pertinax; macroinvertebrados bentônicos; velocidade d'água.

Os simulídeos apresentam hábito hematófago na fase adulta e os imaturos, larvas e pupas, habitam ambientes aquáticos lóticos, onde são encontrados nos locais de maior velocidade do fluxo d'água, em diferentes substratos, como: vegetação ciliar pendente na água, raízes, vegetação aquática, seixos e fundo rochoso (Strieder et al. 2002).

Esses insetos podem afetar o desenvolvimento de uma região porque suas picadas, que além de muito incômodas, podem transmitir agentes causadores de doenças ou causar sérias complicações alérgicas ao homem e aos outros animais, podendo resultar em danos econômicos na pecuária, diminuindo a produção de leite e de carne (Anderson \& Voskuil 1963). No sul do Brasil, Chirostilbia pertinax (Kollar,1832) destaca-se como principal alvo dos programas de controle dos simulídeos, por atacar diretamente o homem e animais domésticos (Ruas Neto \& Silveira 1989; Strieder \& Corseuil 1992; Strieder et al. 2006).

No ambiente aquático, as formas imaturas apresentam altas densidades populacionais, formando uma biomassa considerável, o que representa uma importante fonte de alimento para peixes e para um grande número de invertebrados bentônicos predadores (Crosskey, 1990). Fatores como competição e disponibilidade de substratos, podem estar relacionados com a distribuição das larvas e pupas de simulídeos nos locais de maior velocidade d'água (Ciborowski \& Craig 1991; Crosskey 1990; Disney 1972; Malmqvist \& Sackmann 1996; Moreira et al. 1994).

Malmqvist \& Sackmann (1996) ao observarem o 
comportamento das larvas de simulídeos em relação a diferentes velocidades de água e a presença de predadores potenciais, concluíram que embora o aumento da correnteza diminua a eficiência em capturar alimento, aumentam as chances de sobrevivência pela redução de ataque por predadores. Nesse contexto, o presente trabalho teve como objetivo investigar a relação entre a velocidade de água e a distribuição das formas imaturas de C. pertinax e macroinvertebrados bentônicos associados.

\section{MATERIALE MÉTODOS}

O estudo foi desenvolvido no trecho superior do Rio dos Sinos, no município de Caraá, na região meridional da Serra Geral, no Rio Grande do Sul. Os dados foram levantados no período de 9 de agosto de 1999 a 06 de abril de 2000, em dois córregos de $2^{\mathrm{a}}$ ordem, um de $3^{\mathrm{a}}$ ordem e um de $4^{\mathrm{a}}$ ordem, num percurso de $31 \mathrm{~km}$ ao longo do rio principal, entre as coordenadas geográficas de $29^{\circ} 40^{\prime}$ a $29^{\circ} 70^{\prime}$ de latitude Sul e $50^{\circ} 15^{\prime}$ a $50^{\circ} 40^{\prime}$ de longitude Oeste, onde a vegetação ciliar encontra-se parcialmente preservada e as áreas limítrofes são dominadas por pequenas propriedades, com pecuária e agricultura de subsistência (Fig. 1).

As coletas dos simulídeos e macroinvertebrados associados foram realizadas em quatro datas ao longo do ano, com o objetivo de amostrar os organismos mais representativos dos diferentes trechos num ciclo sazonal completo. Em cada ponto de coleta foram instalados três amostradores de substrato artificial que permaneceram expostos durante 14 dias, nos locais de correnteza de água. O substrato utilizado foi constituído por uma corda de polipropileno branca, com $0,6 \mathrm{~cm}$ de diâmetro e $50 \mathrm{~cm}$ de comprimento. Ao longo dessa corda foram fixadas em distâncias iguais cinco cordas desfiadas e cinco tiras de elástico, ambas com $20 \mathrm{~cm}$ de comprimento. Na extremidade do substrato foi fixado um peso de metal e duas fitas plásticas amarelas, com $4 \mathrm{~cm}$ de largura e $30 \mathrm{~cm}$ de comprimento (Fig. 2).

Antes do recolhimento do substrato artificial foi utilizado um equipamento portátil (Marsh-McBirney Flowmate 2000) para medir a velocidade d'água em três pontos no local da sua instalação. $\mathrm{Na}$ avaliação da distribuição diferenciada dos macroinvertebrados bentônicos, as médias das medidas de velocidade d'água foram agrupadas em três intervalos de igual amplitude: baixa $(0,20$ a $0,63 \mathrm{~m} / \mathrm{s})$, média $(0,64$ a $1,07 \mathrm{~m} / \mathrm{s})$ e alta $(1,08$ a $1,51 \mathrm{~m} / \mathrm{s})$.

Em campo, os substratos com a fauna aderida foram acondicionados em frascos de vidro de $1.000 \mathrm{~mL}$ contendo álcool etílico $96^{\circ} \mathrm{C}$. Em laboratório, o material foi triado e separado em duas categorias: Simuliidae e macroinvertebrados bentônicos associados, sendo acondicionado em frascos com álcool $70 \%$ e incluído na coleção do Laboratório de Entomologia (Universidade do Vale do Rio dos Sinos).

Os macroinvertebrados foram identificados em nível taxonômico de família utilizando as chaves dicotômicas de Merritt \& Cummins (1984), Lopretto \& Tell (1995) e Fernández \& Domínguez (2001) e, com base em bibliografia especializada

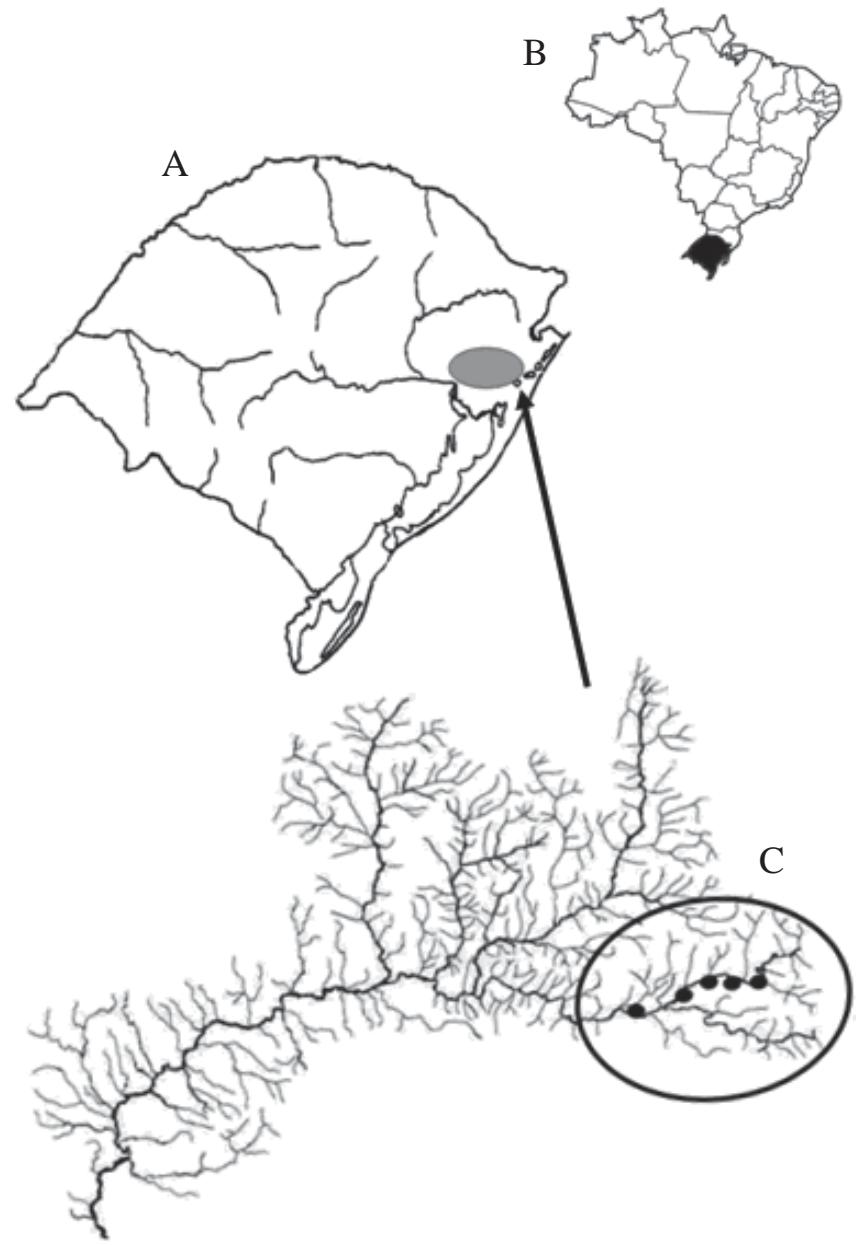

Fig. 1. Estado do Rio Grande do Sul (A), Brasil (B). Localização dos cinco pontos de coleta na bacia hidrográfica do Rio dos Sinos (C).

foi verificado quais das famílias incluem predadores de simulídeos.

As formas imaturas de simulídeos foram divididas em três categorias: larvas de ínstares iniciais, larvas do último ínstar (caracterizadas pela presença de histoblasto branquial bem desenvolvido) e pupas. As larvas do último ínstar e as pupas de $C$. pertinax foram identificadas e separadas das demais espécies. No presente trabalho seguiu-se a posição taxonômica que propõem a elevação dos subgêneros ao nível de gênero em Simuliidae Newman, 1834, conforme a amplitude e nomenclatura usada por Py-Daniel \& Moreira Sampaio (1994).

Para as análises estatísticas os dados de abundância total foram log transformados, removendo assim a heteroscedasticidade da amostra. Para avaliar a variação da abundância total dos grupos de organismos coletados nas diferentes classes de velocidade d'água (baixa, média e alta) foi realizado ANOVA One-Way, com teste post-hoc de Tukey. Para avaliar a influência da velocidade da água e da fauna associada sobre a abundância de $C$. pertinax foi realizada uma regressão múltipla. Os testes foram realizados no programa Systat (2004) versão 11. 


\section{RESULTADOS EDISCUSSÃO}

Foram coletados 39.598 organismos bentônicos, sendo 33.418 simulídeos e 6.180 macroinvertebrados associados. Entre os simulídeos, 27.714 espécimes correspondem aos ínstares larvais iniciais e 5.704 a larvas do último ínstar e pupas, sendo destes últimos estágios 828 da espécie C. pertinax. Os macroinvertebrados coletados pertencem a 20 famílias de insetos, duas famílias de crustáceos e uma família de gastrópodes (Tabela I).

O aumento da velocidade d'água demonstrou uma redução na abundância de macroinvertebrados. No global, 61,5\% dos indivíduos foram coletados na classe de velocidade baixa $(0,20$ a $0,63 \mathrm{~m} / \mathrm{s}), 27,1 \%$ na velocidade média $(0,64$ a $1,07 \mathrm{~m} / \mathrm{s})$ e $11,4 \%$ na velocidade alta $(1,08$ a $1,50 \mathrm{~m} / \mathrm{s})$. Houve também uma redução no número de famílias com o aumento da velocidade de água. Na classe de velocidade baixa ocorreram 22 famílias, seguido pela velocidade média, com 15 famílias, e velocidade alta com apenas 13 .

Dentre os macroinvertebrados existem vários predadores das formas imaturas de simulídeos, conforme os diversos autores relacionados na Tabela II. As famílias com predadores de simulídeos sofreram uma redução na abundância com o aumento da velocidade d'água. Na velocidade baixa ocorreram 3.139 indivíduos $(62,4 \%)$, pertencentes a onze famílias; seguido pela velocidade média com 1.210 indivíduos $(24,1 \%)$, pertencentes a nove famílias; e velocidade alta com 681 indivíduos (13,5\%), incluídos em oito famílias. Esses resultados estão de acordo com Malmqvist \& Sackmann (1996), que afirmam que as altas correntezas proporcionam um ambiente desfavorável para a ação de predadores.
Tabela I. Macroinvertebrados associados aos imaturos de simulídeos, coletados utilizando-se substrato artificial, no trecho superior do Rio dos Sinos, Rio Grande do Sul.

\begin{tabular}{|c|c|c|c|c|c|}
\hline \multirow{2}{*}{$\begin{array}{l}\text { Ordem } \\
\text { Plecoptera }\end{array}$} & \multirow{2}{*}{$\begin{array}{l}\text { Família } \\
\text { Perlidae }\end{array}$} & \multicolumn{3}{|c|}{$\begin{array}{l}\text { Velocidade d'água } \\
\text { Baixa Média Alta }\end{array}$} & Total \\
\hline & & 70 & 21 & 9 & $100(1,62 \%)$ \\
\hline & Gripopterygidae & 583 & 440 & 19 & $1.042(16,92 \%)$ \\
\hline \multirow[t]{6}{*}{ Trichoptera } & Hydropsychidae & 757 & 354 & 315 & $1.426(23,15 \%)$ \\
\hline & Hydroptilidae & 0 & 0 & 1 & $01(0,02 \%)$ \\
\hline & Hydrobiosidae & 20 & 24 & 6 & $50(0,81 \%)$ \\
\hline & Leptoceridae & 3 & 4 & 0 & $07(0,11 \%)$ \\
\hline & Calamoceratidae & 0 & 1 & 0 & $01(0,02 \%)$ \\
\hline & Sericostomatidae & 24 & 13 & 0 & $37(0,60 \%)$ \\
\hline \multirow[t]{3}{*}{ Diptera } & Chironomidae & 1.270 & 426 & 207 & $1.903(30,89 \%)$ \\
\hline & Blephariceridae & 1 & 1 & 0 & $02(0,03 \%)$ \\
\hline & Psychodidae & 0 & 0 & 01 & $01(0,02 \%)$ \\
\hline \multirow[t]{3}{*}{ Ephemeroptera } & Baetidae & 836 & 304 & 103 & $1.243(20,18 \%)$ \\
\hline & Leptohyphidae & 37 & 10 & 15 & $62(1 \%)$ \\
\hline & Leptophlebiidae & 3 & 0 & 2 & $05(0,08 \%)$ \\
\hline \multirow[t]{2}{*}{ Coleoptera } & Elmidae & 133 & 61 & 20 & $214(3,47 \%)$ \\
\hline & Gyrinidae & 1 & 0 & 0 & $01(0,02 \%)$ \\
\hline Hemiptera & Naucoridae & 3 & 0 & 0 & $03(0,05 \%)$ \\
\hline \multirow{2}{*}{ Odonata } & Calopterygidae & 12 & 0 & 0 & $12(0,19 \%)$ \\
\hline & Libellulidae & 1 & 1 & 0 & $02(0,03 \%)$ \\
\hline Megaloptera & Corydalidae & 1 & 0 & 0 & $01(0,02 \%)$ \\
\hline Amphipoda & Hyalellidae & 2 & 1 & 0 & $03(0,05 \%)$ \\
\hline Decapoda & Aeglidae & 1 & 0 & 0 & $01(0,02 \%)$ \\
\hline Mesogastropoda & Hydrobiidae & 40 & 0 & 3 & $43(0,70 \%)$ \\
\hline Total & & 3.798 & 1661 & 701 & 6.160 \\
\hline
\end{tabular}

Total $=$ somatórios das réplicas realizadas em três classes de velocidade da água: Baixa $(0,20$ a $0,63 \mathrm{~m} / \mathrm{s})$, Média $(0,64$ a $1,07 \mathrm{~m} / \mathrm{s})$, Alta $(1,08$ a $1,50 \mathrm{~m} / \mathrm{s}$ ) no período de maio de 1999 a abril de 2000 .

Tabela II. Macroinvertebrados coletados no trecho superior do Rio dos Sinos, e que incluem predadores de Simuliidae.

\begin{tabular}{|c|c|c|c|}
\hline Ordem & Família & Estágios predados & Referências bibliográficas \\
\hline Plecoptera & Perlidae & Le P & $\begin{array}{l}\text { Castaldi \& Gutiérrez 1981; Crisp 1956; Crosskey 1990; Elliott 2000; Gorayeb \& } \\
\text { Mok 1982; Gorayeb \& Pinger 1978; Jenkins 1964; Laird 1981; Wotton et al. } \\
1993 .\end{array}$ \\
\hline \multirow[t]{4}{*}{ Trichoptera } & Hydropsychidae & $\mathrm{L}$ & $\begin{array}{l}\text { Crosskey 1990; Damalt 1956; Gorayeb \& Pinger 1978; Jenkins 1964; Kuralová \& } \\
\text { Olejnícek 1985; Laird 1981; Malmqvist 1994; Service } 1974 .\end{array}$ \\
\hline & Hydroptilidae & $\mathrm{Pe} \mathrm{Ov}$ & Crosskey 1990; Disney 1972. \\
\hline & Hydrobiosidae & $\mathrm{L}$ & Flint 1983. \\
\hline & Leptoceridae & $\mathrm{L}$ & Crisp 1956. \\
\hline Diptera & Chironomidae & Ov & $\begin{array}{l}\text { Crisp 1956; Crosskey 1990; Jenkins 1964; Laird 1981; Lindegaard 1997; } \\
\text { Peterson \& Davies 1960; Werner \& Pont } 2003 .\end{array}$ \\
\hline \multirow[t]{2}{*}{ Ephemeroptera } & Baetidae & Le P & Crisp 1956; Service 1974; Crosskey 1990. \\
\hline & Leptohyphidae & L e P & Crosskey 1990. \\
\hline Coleoptera & Elmidae & $\mathrm{L}$ & Crosskey 1990; Jenkins 1964; Gorayeb \& Pinger 1978; Gorayeb \& Mok 1982. \\
\hline Odonata & Libellulidae & Le P & Crisp 1956; Crosskey 1990; Jenkins 1964; Gorayeb \& Pinger 1978; Service 1974. \\
\hline Megaloptera & Corydalidae & Le P & $\begin{array}{l}\text { Crosskey 1990; Damalt 1956; Flint 1973; Gorayeb \& Pinger 1978; Laird 1981; } \\
\text { Gorayeb \& Mok } 1982 .\end{array}$ \\
\hline Decapoda & Aeglidae & Le P & Magni \& Py-Daniel 1989. \\
\hline
\end{tabular}

Ov: ovo L: larva P: pupa 


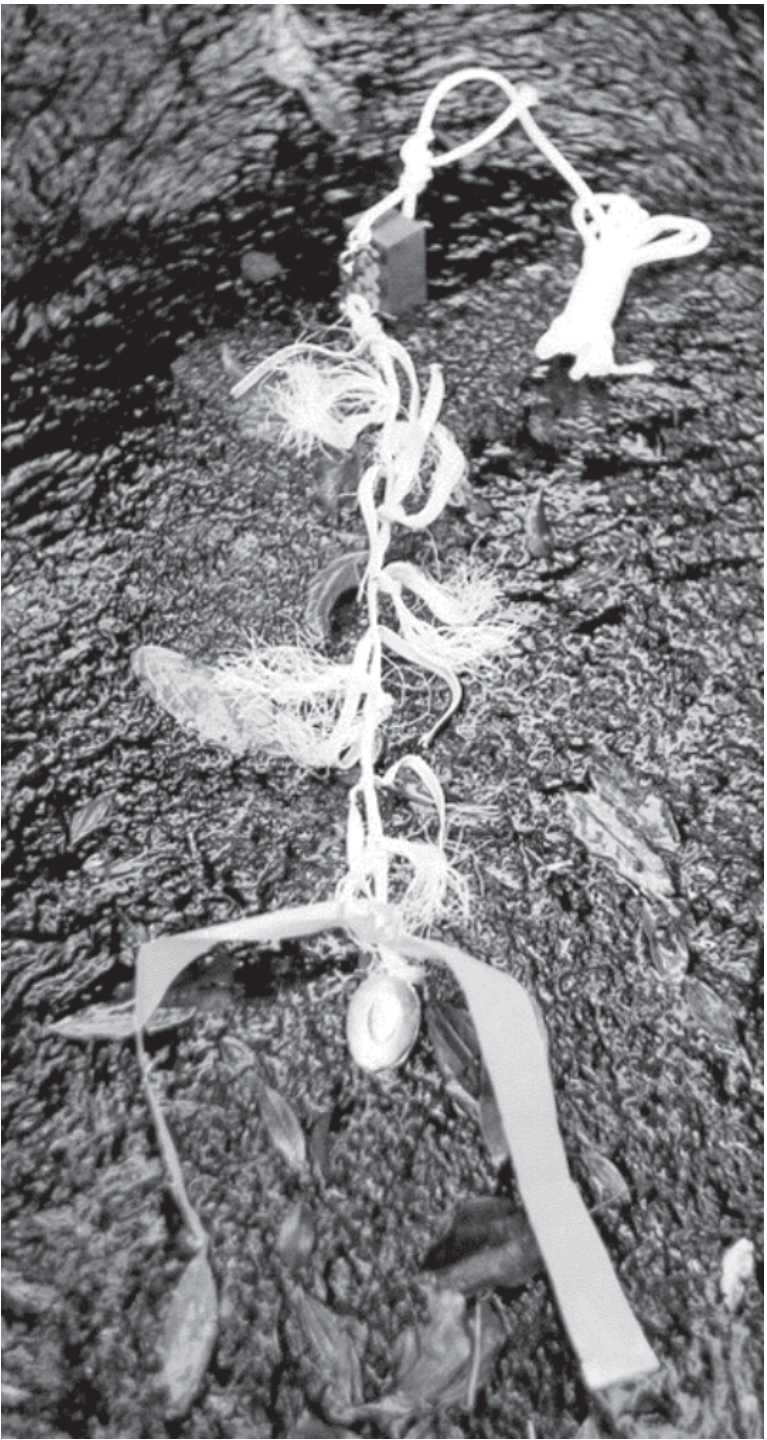

Fig. 2. Substrato utilizado na coleta de simulídeos e dos macroinvertebrados associados no curso superior da bacia do Rio dos Sinos, Rio Grande do Sul.

Magni \& Py-Daniel (1989) em observações realizadas na bacia hidrográfica do alto rio Uruguai, no extremo norte do estado do Rio Grande do Sul, verificaram que as formas maiores dos caranguejos do gênero Aegla ocorrem em locais com velocidades reduzidas da água e apenas os indivíduos jovens foram observados nos locais com grande concentração de imaturos de simulídeos.

No presente trabalho foi verificado que o total das formas imaturas de simulídeos seguiu o mesmo padrão de distribuição dos macroinvertebrados associados (Tabela III). A ANOVA mostrou que a abundância dos grupos estudados foi similar entre as diferentes categorias de velocidade d'água, sendo para $C$. pertinax $\left(\mathrm{F}_{250}=0,019 ; \mathrm{P}=0,982\right)$, macroinvertebrados $\left(\mathrm{F}_{2,50}=0,634 ; \mathrm{P}=0,535\right)$, famílias que incluem predadores de Simuliidae $\left(\mathrm{F}_{2.50}=0,410 ; \mathrm{P}=0,666\right)$ e total de Simuliidae $\left(\mathrm{F}_{2.50}\right.$ $=1,027 ; \mathrm{P}=0,366$ ).

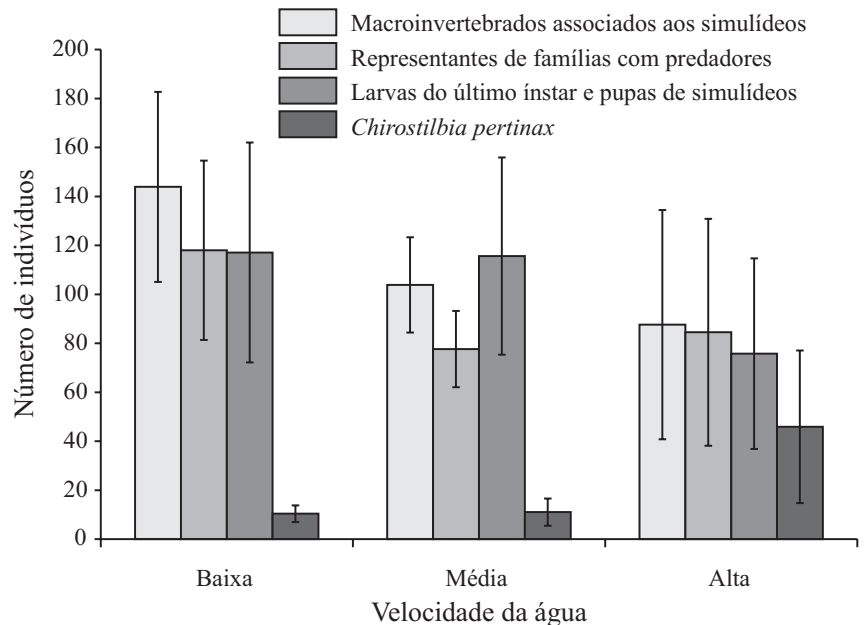

Fig. 3. Número médio de organismos coletados em três classes de velocidade d'água: baixa $(0,20$ a $0,63 \mathrm{~m} / \mathrm{s})$, média $(0,64$ a $1,07 \mathrm{~m} / \mathrm{s})$ e alta $(1,08$ a $1,50 \mathrm{~m} / \mathrm{s})$, utilizando-se substrato artificial, no trecho superior do Rio dos Sinos, Rio Grande do Sul. As barras indicam o desvio padrão.

C. pertinax demonstrou uma distribuição diferenciada em relação ao total de simulídeos (Fig. 3), na menor velocidade da água foram encontrados 255 indivíduos (30,8\%), na velocidade média $206(24,9 \%)$ e na alta $367(44,3 \%)$. Essa espécie corresponde a $60,6 \%$ das larvas no último ínstar e pupas coletadas na velocidade alta. Segundo Strieder et al. (2006), a distribuição local e os níveis populacionais das formas imaturas dos simulídeos estão diretamente relacionados aos fatores ecológicos que atuam de forma inter-relacionada e interdependente em uma determinada bacia hidrográfica. Desequilíbrios nesses fatores podem alterar a diversidade biológica e favorecer a proliferação de determinadas espécies, levando as indesejáveis infestações das formas adultas com hábito hematófago.

A abundância de C. petinax aumenta à medida que os macroinvertebrados predadores diminuem e os não predadores aumentam $\left(\mathrm{GLM}, \mathrm{R}^{2}=0,207 ; \mathrm{F}_{2.50}=6,536 ; \mathrm{P}=0,003\right)$, revelando uma tendência em ocupar microhabitat com menor presença de predadores. Estes resultados estão de acordo com Strieder et al. (2002), quando afirmam que os diferentes padrões de distribuição das formas imaturas dos simulídeos, podem ser

Tabela III. Organismos bentônicos coletados no trecho superior do Rio dos Sinos e sua distribuição em três classes de velocidade d'água.

\begin{tabular}{lcccc}
\hline \multirow{2}{*}{ Táxons } & \multicolumn{3}{c}{ Velocidade d'água } & \multirow{2}{*}{ Total } \\
& Baixa & Média & Alta & \\
\hline Simuliidae $(\mathrm{Li}+\mathrm{Lu}+\mathrm{P})$ & 19.578 & 8.434 & 5.406 & 33.418 \\
Simuliidae $(\mathrm{Lu}+\mathrm{P})$ & 3.513 & 1.585 & 606 & 5.704 \\
C. pertinax $(\mathrm{Lu}+\mathrm{P})$ & 255 & 206 & 367 & 828 \\
Famílias predadoras de Simuliidae & 11 & 09 & 08 & 12 \\
Indivíduos predadores de Simuliidae & 3.139 & 1.210 & 681 & 5.030 \\
\hline
\end{tabular}

Li: Larvas nos ínstares iniciais; Lu: Larvas no último ínstar; P: pupas 
explicados pelos processos evolutivos das biocenoses na dependência direta dos recursos espaciais disponíveis, sendo alcançado pela distribuição diferenciada das espécies um melhor aproveitamento dos micro-habitats no ambiente aquático.

Agradecimentos. A Manuel Christoph Strauch e Pe. Edward Benya, SJ pela revisão do abstract. À Cristina Stenert pelo auxílio nas análises estatísticas. À FAPERGS pela bolsa de iniciação científica ao primeiro autor e aos colegas do laboratório de Entomologia da UNISINOS pela colaboração na realização desse trabalho.

\section{REFERÊNCIAS}

Anderson, R. \& G. H. Voskuil. 1963. A reduction in milk production caused by feeding of black flies (Diptera: Simuliidae) on dairy cattle in California, with notes on the feeding activity on other animals. Mosquito News 23: 126-131.

Castaldi, M. O. \& M. O. Gutiérrez. 1981. Contribución al estúdio de la bioecologia de los simulidos (Diptera: Simuliidae) transmisores de oncocercosis: Entomofauna asociada a las formas larvarias. Folia Entomologica Mexicana 50: 97-107.

Cibrowski, J. J. H. \& D. A. Craig. 1991. Factors influencing dispersion of larval black flies (Diptera: Simuliidae): effects of the presence of an invertebrate predator. Canadian Journal of Zoology 69: 1120-1123.

Crisp, G. 1956. An ephemeral fauna of torrents in the northern territories of the gold coast, with special reference to the enemies of Simulium. British Empire Society for the Blind p. 260-267.

Crosskey, R. W. 1990. The natural history of blackflies. John Wiley \& Sons Ltda. England. $711 \mathrm{p}$.

Dalmat, H. T. 1956. The black flies (Diptera: Simuliidae) of Guatemala and their role as vector of onchocerciasis. Smithsonian Miscellaneous Collections 125: 1-425.

Disney, R. H. L. 1972. Larval Hydroptilidae (Trichoptera) that prey upon Simuliidae (Diptera) in Cameroon. Entomologist's Monthly Magazine 108: 84-85.

Elliott, J. M. 2000. Contrasting diel activity and feeding patterns of four species of carnivorous stoneflies. Ecological Entomology 25: $26-34$.

Flint, O. S. 1973. The Megaloptera of Chile. Revista Chilena de Entomologia 7: 31-45.

Flint, O. S. 1983. Studies of Neotropical caddisflies, XXXIII: new species from Austral South America (Trichoptera). Smithsonian Contributions to Zoology 377: 1-100.

Fernández, H. R. \& E. Domínguez (eds). 2001. Guía para la Determinación de los Artrópodos Bentónicos Sudamericanos. Tucumán: Universidad Nacional de Tucumán. 282 p.

Gorayeb, I. S. \& W. Y. Mok. 1982. Comparision of capillary tube and immunodiffusion preciptin test in the detection of Simulium fulvinotum larval predators. Ciência e Cultura 34: 1662-1668.

Gorayeb, I. S. \& R. R. Pinger. 1978. Detecção de predadores naturais das larvas de Simulium fulvinotum Cerqueira e Mello, 1968 (Diptera: Nematocera). Acta Amazonica 8: 629-637.

Jenkins, D. W. 1964. Pathogens, parasites and predators of medically important arthropods, Annoted list and bibliography. WHO. Geneve. 97 p.

Kuralová, M. \& J. Olejnícek. 1985. Predaceous larvae of caddisflies
(Trichoptera) as natural enemies of blackfly larvae (Diptera: Simuliidae) under conditions of South Bohemia. Folia Parasitologica 32: 375-376.

Laird, M. 1981. Blackflies. The future biological methods in integrated control. Academic Press. London. 397 p.

Lindegaard, C. 1997. Diptera Chironomidae, non-biting midges. Volume 2: 265-294. In: Aquatic Insects of North Europe, A Taxonomic Handbook (A. N. Nilsson). Apollo Books. Stenstrup. 440 p.

Lopretto, E. C. \& G. TELL. 1995. Ecosistemas de Aguas Continentales. Metodologias para su estudio (vol. III). Ediciones Sur, La Plata, p. 897-1401.

Magni, S. T. \& V. Py-Daniel. 1989. Aegla platensis Schmitt, 1942 (Decapoda: Anomura) um predador de imaturos de Simuliidae (Diptera: Culicomorpha). Revista de Saúde Pública 23: 258 259.

Malmqvist, B. 1994. Preimaginal blackflies (Diptera: Simuliidae) and their predators in a central Scandinavian Lake outlet stream. Annales Zoologici Fennici 31: 245-255.

Malmqvist, B. \& G. Sackmann. 1996. Changing risk of predation for a filter-feeding insect along a current velocity gradient. Oecologia 108: $450-458$.

Merritt, R. W. \& K. W. Cummins (eds). 1984. An introduction to the aquatic insects of North America ( $2^{\text {nd }}$ ed.). Kendall/ Hunt Publ. Dubuque, xiii $+722 \mathrm{p}$.

Moreira, G. R. P.; R. A. Pegoraro \& G. Sato. 1994. Influência de fatores abióticos sobre o desenvolvimento de Simulium nogueirai D’Andreatta \& González em um córrego da mata Atlântica. Anais da Sociedade Entomológica do Brasil 23: 525-542.

Peterson, B. V. \& D. M. Davies. 1960. Observations on some insects predators of blak flies (Diptera: Simuliidae) of Algonquim Park, Ontario. Canadian Journal of Zoology 38: 8-9.

Py-Daniel, V. \& R. T. Moreira Sampaio. 1994. Jalacimgomyia gen.n. (Culicomorpha); a ressurreição de Gymnopaidinae; a eliminação do nível tribal; apresentação de novos caracteres e a redescrição dos estágios larval e pupal de Simulium colombaschense (Fabricius, 1787) (Diptera: Simuliidae). Memorias del Centro Amazónico para Investigación y Control de Enfermedades Tropicales CAICET 4: 101-148.

Ruas Neto, A. L. \& S. M. Silveira. 1989. Uso de inseticidas bacterianos para o controle de culicídeos e simulídeos no Rio Grande do Sul. Memórias do Instituto Oswaldo Cruz 84: 39-45.

Service, M. W. 1974. Detection of the predators of Simulium damnosum by the precipitin test. Annals of Tropical Medicine and Parasitology 69: 105-108.

Strieder, M. N. \& E. Corseuil. 1992. Atividades de hematofagia em Simuliidae (Diptera, Nematocera) na Picada Verão, Sapiranga, RS Brasil. Acta Biologica Leopoldensia 14: 75-98.

Strieder, M. N.; J. E. Santos Jr. \& A. M. O. Pes. 2002. Diversidade e distribuição de Simuliidae (Diptera: Nematocera) no gradiente longitudinal da bacia do Rio dos Sinos, no Rio Grande do Sul, Brasil. Entomología y Vectores 9: 527-540.

Strieder, M. N.; J. E. Santos Jr. \& E. M. Vieira. 2006. Distribuição, abundância e diversidade de Simuliidae (Diptera, Nematocera) em uma bacia hidrográfica impactada no Sul do Brasil. Revista Brasileira de Entomologia 50: 119-124.

Systat. 2004. Systat for Windows Versão 11. Systat Software Inc.

Werner, D. \& A. C. Pont. 2003. Dipteran predators of Simuliid blackflies: a worldwide review. Medical and Veterinary Entomology 17: 115-132.

Wotton. R. S.; M. S. Wipfli; L. Watson \& R. W. Merritt. 1993. Feeding variability among individual aquatic predators in exptal channels. Canadian Journal of Zoology 71: 2033-2037.

Recebido em 20/03/2006; aceito em 21/12/2006 